\title{
LOS DESLINDES DE HEREDADES DE SEPÚLVEDA (SIGLO XV): ESTUDIO DIPLOMÁTICO
}

\author{
Carlos SÁEZ \\ Universidad de Alcalá de Henares \\ ANTONIO CASTILLO \\ Universidad Complutense de Madrid
}

El apeo o deslinde de heredades es un tipo documental de pleno valor jurídico y diplomático que hasta hace poco había sido completamente ignorado por manuales y especialistas de la materia. Según la definición del Diccionario de la Real Academia Española, el apeo es un «instrumento jurídico que acredita el deslinde y demarcación" de propiedades. Su doble carácter de documento jurídicamente público y de documento fiscal (aunque este aspecto no siempre se descubra en el tenor documental) relaciona en cierto sentido al apeo con un grupo bastante numeroso de documentos. ${ }^{1}$

Parece que los antecedentes más antiguos del apeo como documento diplomático se remiten al menos hasta el siglo XI. Desde entonces, la delimitación y adjudicación de términos de heredades a sus poseedores o beneficiarios es una facultad real ejercida en el ámbito de la administración de justicia. Así lo acreditan diversos documentos fechados en esa época. ${ }^{2}$ Nosotros abordaremos en nuestro trabajo ejemplos más tardíos procedentes de los fondos del siglo XV de la villa de Sepúlveda, que hoy se encuentran en su mayoría en la sección de Clero del Archivo Histórico Nacional.

El apeo más antiguo conservado en estos fondos data de $1413^{3}$. Sin embargo, para la confección de este trabajo hemos elegido otro posterior, de

1 Reseñados por Santos García Larragueta, El apeo, documento diplomático, "Anuario de Estudios Medievales" 17 (1988), pp. 617-618: políptico; estado general de bienes y censos eclesiásticos; libros con carácter de registros territoriales, caso del censier, terrier, liève o de los rentiers, etc.

${ }^{2}$ Ibidem, pp. 618-619.

${ }^{3}$ Fue editado por Carlos SÁzZ, Colección Diplomática de Sepúlveda, II, Segovia 1991, docs. 65 y ss. 
$1452^{4}$, puesto que se trata del caso más completo que hemos hallado. En él concurren un total de siete documentos, que son todos los que constituyen los deslindes sepulvedanos. Los demás casos que conocemos son incompletos pues alguno de dichos documentos falta siempre.

La potestad de apear territorios no fue exclusiva del monarca. Del siglo XII datan algunos testimonios que demuestran que también podían ejercerla la autoridad señorial, eclesiástica, concejil o notarial. Esta es la causa de la gran variedad de formas diplomáticas y procesos de redacción que adoptaron estos documentos.'

La elaboración del apeo se inicia a partir de una pesquisa, que da lugar a una serie de pruebas testificales y documentales que se incorporan a la documentación procesal redactada normalmente por un notario público, incluso en el caso de aquellos apeos realizados por autoridades eclesiásticas. Los testimonios para realizar el apeo pueden ser tanto escritos -libros y documentos antiguos-como orales - declaraciones de hombres buenos ${ }^{6}$ conocedores de las propiedades rústicas y de sus términos-.

Desde muy antiguo existen apeos redactados sin un formulario definido y sólo a partir de los siglos XIV y XV puede decirse que la redacción de estos documentos sigue unas pautas más uniformes que permiten hablar de la consolidación de una fórmula diplomática visible. ${ }^{7}$ En nuestro caso, el formulario varía muy poco desde el primer ejemplo aludido de 1413 que, por otra parte, parece ser un caso ya totalmente canonizado debido a su compleja redacción formularia y composición documental. Que no tengamos ejemplos anteriores entre los fondos sepulvedanos se debe únicamente a la fatalidad de la pérdida de documentación.

En nuestro apeo, un vecino de Sepúlveda desea deslindar sus propieda-

${ }^{4}$ Ibidem, docs. 105 a 111.

'Garcia larragueta, ob. cit., suministra numerosos ejemplos y divide los apeos en resultantes de concordias, pactos o avenencias, de propiedades de señoríos eclesiásticos y realizados con intervención de la autoridad civil. Un caso en extremo complejo fue descrito por el mismo autor en Fuero de Almaraz al monasterio de Valparaíso, "Archivos Leoneses" (1988), pp. 83-104. Debemos otros dos estudios diplomáticos sobre los deslindes de heredades a Manuel Larriba Baciero: Los apeos de Alialá de Henares: Estudio diplomático, en Actas del III Encuentro de Historiadores del Valle del Henares, Guadalajara 1992, págs. 123-145; Apeos y deslindes entre Alcalá de Henares y Torrejón de Ardoz (siglos XVI-XIX), en Actas del III Encuentro (cit.), págs. 323-332.

${ }^{6}$ Los llamados apeadores en la documentación sepulvedana.

7 Al respecto nos remitimos a Garcia Larragueta, El apeo (cit)., pp. 630-633 y Fuero de Almaraz (cit.), págs. 95-104, donde se estudian más detenidamente las particularidades de las distintas diligencias practicadas al elaborar el documento de apeo. Sin embargo, notamos que entre ambos procesos hay diferencias, como ocurre con el ejemplo que proponemos aquí. 
des ${ }^{8}$ situadas en varios pueblos del alfoz y para ello eleva una petición a la autoridad. En este punto encontramos un hecho interesante pues al inicio del proceso esta autoridad es señorial mientras que al final del mismo es real, como más adelante comentaremos. Veamos cual es el proceso de redacción de los deslindes sepulvedanos".

\section{DOCUMEnTO 1}

El primer documento del proceso de los deslindes consiste, por tanto, en una petición, por parte del interesado, que comparece personalmente, a la máxima autoridad local, en este caso un alcalde de la villa nombrado por el corregidor de la misma ${ }^{10}$ para llevar a cabo el apeo y para dar los oportunos pregones. La petición culmina en el expositivo con la concesión de licencia para ambos actos y con un mandato para realizar los pregones según la costumbre de la villa.

Recogemos a continuación, como haremos con todos los documentos del deslinde, fecha, regesto y estructura diplomática de este primer diploma. ${ }^{11}$

\section{2, mayo 4, Sepúlveda}

Juan Esquerra de Angulo, bachiller y alcalde de Sepúlveda por el corregidor Sancho de Velasco, concede licencia a Alvar Rodríguez de Vellosillo, vecino de Sepúlveda, para deslindar las heredades que posee en Rebollar, Valdesimonte, Aldea el Pozo, Fresnillo de la Fuente, Bercimuel, Pajarejos, Grajera. Castillejo, Aldealapeña, Sigueruelo, Casla y Blasco Miguel, aldeas de Sepúlveda, y para dar los correspondientes pregones de la forma acostumbrada en la villa.

${ }^{8}$ En total 117, según el documento 111 de los citados en la nota 4. En el doc. 68 del segundo volumen de la Colección Diplomática (cit.), se menciona un motivo particular por el cual se confecciona un apeo: los clérigos del cabildo de Sepúlveda poseían una heredad en la aldea de Las Covachuelas pero non sabian toda a dicha heredad e que se les perdía e enagenava, $e$ aun que algunos ommes que ge la entravan e labravan non devidamente. En este caso, la autoridad que preside el deslinde es eclesiástica.

${ }_{9}$ El sistema que vamos a describir coincide con el empleado por la Casa de la Caridad, institución benéfico municipal que deslindó todos sus términos en la segunda mitad del XV. Ver al respecto el trabajo de Carlos Saez, La Casa de Caridad de Sepúlveda. Propiedades en la primera mitad del siglo XV, en Actas del I Congreso de Historia de Castilla y León, Burgos 1983, págs. 371-386.

${ }_{10}$ Nombrado a su vez por quien ostentaba en aquel momento el señorío de la villa, el maestre de Santiago y condestable de Castilla, don Álvaro de Luna, es decir una autoridad señorial, como se ha anunciado.

$"$ En los siete documentos figuran una serie de fórmulas habituales que no comentaremos, como la presencia del escribano y testigos y la relación nominal de estos últimos. 
CDS II, 105.

\section{Data}

En la villa de Sepúlvega, jueves quatro días del mes de mayo, año del Nasçimiento del nuestro Salvador Ihesuchristo de mill e quatroçientos e çincuenta e dos años,

\section{Presencia de la autoridad}

antel bachiller Juan Esquerra de Angulo, alcallde en la dicha villa e su tierra por el honrado cavallero Sancho de Velasco, corregidor en la dicha villa e su tierra por nuestro señor el maestre de Santiago e condestable de Castilla,

\section{Presencia de escribano-testigos}

e en presençia de mí Pero Gonçález, escrivano público por el conçejo en la dicha Sepúlvega, e de los testigos de yuso escritos,

\section{Comparecencia} villa,

paresçió y presente Alvar Rodríguez de Vellosillo, vezino de la dicha

\section{Exposición}

e dixo que por quanto él quería apear çiertas heredades de pan levar e de non levar, e prados, e pastos, e casas, e heras, e solares que él tiene en Rebollar, e en Valdesimonte, e en Aldea el Pozo, e en Fresnillo, e en Verzimuel, e en Pajarejos, e en Grajera, e en Castillejo, e en Aldealapeña, e en Sigueruelo, e en Casla, e en Blasco Miguel, aldeas e término de la dicha villa, e en sus devisas e labranças,

\section{Partícula de unión por ende,}

\section{Súplica 1}

dixo que pedía e pidió al dicho alcallde que le dé licencia para apear las dichas heredades e cada una dellas,

\section{Súplica 2} villa.

e ansimesmo para dar los pregones segund forma e horden de la dicha

\section{Disposición 1}

E luego el dicho alcallde dixo que oía lo que dezía e que le dava e dio la dicha liçençia para apear las dichas heredades e cada una dellas, 


\section{Disposición 2}

ansimesmo para dar los dichos pregones en quanto podía e de derecho devía,

\section{Fórmula de perjuicio} oviese,

non parando perjuizio a ninguna persona que algund derecho a ello

\section{Mandato}

e que mandava e mandó fazer los dichos pregones, segund la forma e horden acostumbrada desta dicha villa.

\section{Testigos}

Testigos que a esto fueron presentes: Alfonso Núñez, escrivano, e Diego Gonçález de Valençia, e Juan de Peñaranda, e Pero, criado del dicho alcallde, e otros.

\section{Diferencia de tiempo con el siguiente documento \\ 0 días}

\section{DOCUMENTO 2}

El segundo documento consiste en la promulgación de un primer pregón, que tiene lugar en el arrabal de la villa ${ }^{12}$ por mandato de la autoridad y a petición del interesado. El pregón es publicado a altas bozes por el pregonero público de la villa y en él se anuncian el deslinde y los lugares donde se va a efectuar, se advierte a los posibles interesados en el mismo (todos los que son surqueros) para que lo vayan a ver apear por que después non pretendan inorançia, y, por último, se indica el número del pregón.

\section{[1452, mayo 4], Sepúlveda}

Frutos Sánchez, pregonero público de Sepúlveda, a petición de Alvar Rodríguez de Vellosillo, vecino de Sepúlveda, hace saber, en primer pregón, a los que tienen beredades en Rebollar, Valdesimonte, Aldea el Pozo, Fresnillo de la Fuente, Bercimuel, Grajera, Pajarejos, Castillejo, Aldealapeña, Sigueruelo, Casla y Velasco Miguel, aldeas de Sepúlveda, que se va a proceder al deslinde de las posesiones del citado Alvar Rodríguez en dichos lugares.

12 Otro lugar en el que tenían lugar los pregones era la plaza de la villa. En ocasiones, los pregones del arrabal se sitúan en un lugar más concreto: en la peñuela del arrabal. 
CDS II, 106

1. Particula de unión con el documento anterior

E después desto,

2. Lugar del pregón

en el arraval de la dicha Sepúlvega,

3. Data

este dicho día e mes e año susodicho,

4. Presencia escribano-testigos

en presençia de mí el dicho escrivano e de los testigos yuso escritos,

5. "Iussio-rogatio"

por mandado del dicho alcallde e a pedimiento del dicho Alvar Rodríguez,

6. Anuncio del pregón

por Frutos Sánchez, pregonero público en la dicha villa, fue dado el primero pregón a altas bozes en esta guisa:

7. Notificación

Sepan todos

\section{Anuncio del propietario}

que Alvar Rodríguez de Vellosillo, vezino de la dicha villa,

\section{Anuncio de deslinde}

que quiere apear todas las heredades de pan levar e de non levar, e prados, e pastos, e heras, e casas, e solares quel dicho Alvar Rodríguez ha e tiene en Rebollar, en Valdesimonte, e en Aldea el Pozo, e en Fresnillo de la Fuente, e en Verzimuel, e en Grajera, e en Pajarejos, e Castillejo, e en Aldealapeña, e en Sigueruelo, e Casla, e Velasco Miguel, aldeas e término de la dicha Sepúlvega, e en sus devisas e labranças.

\section{Partícula de unión}

Por ende,

\section{Citación a los interesados}

que todos los que son surqueros de las dichas heredades, que lo vayan a ver apear por que después non pretendan inorançia, 
12. Anuncio del número de pregón

que este es el primero pregón.

\section{Testigos}

Testigos que a esto fueron presentes: Mate Sánchez de Sobrepeña, vezino del Villar, e Andrés Gonçález, vezino de Castroximeno, e Juan, fijo de Asenxo Martín, vezino de Aldealcorvo, aldeas de la dicha Sepúlvega.

14. Diferencia de tiempo con el siguiente documento

7 días

\section{DOCUMENTO 3}

Siete días después, se promulga el segundo pregón. Sus características son idénticas a las del anterior.

1452, mayo 11, Sepúlveda

Frutos Sánchez, pregonero público de Sepúlveda, a petición de Alvar Rodríguez de Vellosillo, vecino de Sepúlveda, hace saber, en segundo pregón, a los que tienen heredades en Rebollar, Valdesimonte, Aldea el Pozo, Fresnillo de la Fuente, Bercimuel, Pajarejos, Grajera, Castillejo, Aldealapeña, Sigueruelo, Casla y Velasco Miguel, aldeas de Sepúlveda, que se va a proceder al deslinde de las posesiones del citado Alvar Rodríguez en dichos lugares.

CDS II, 107.

\section{Partícula de unión con documento anterior}

E después desto,

\section{Lugar del pregón}

en dicho arraval de la dicha Sepúlvega,

\section{Data}

jueves honze días del mes de mayo, año susodicho,

\section{Presencia escribano-testigos}

e en presençia de mí el dicho Pero Gonçález, escrivano público sobredicho, e de los testigos de yuso escritos,

\section{5. «Iussio-rogatio»}

por mandado del dicho alcallde e a pedimiento del dicho Alvar Rodríguez de Vellosillo, 


\section{Anunizo del pregón}

por el dicho Frutos Sánchez, pregonero público en la dicha villa, fue dado el segundo pregón a altas bozes en esta guisa:

\section{Notificación}

Sepan todos

\section{Anuncio del propietario}

quel dicho Alvar Rodríguez de Vellosillo, vezino de la dicha villa,

\section{Anuncio del deslinde}

que quiere apear todas las heredades que él ha e tiene en Rebollar, e en Valdesimonte, en Aldea el Pozo, e en Fresnillo, e en Verzimuel, e en Pajarejos, e en Grajera, e en Castillejo, e en Aldealapeña, e en Sigueruelo, e en Casla, e en Velasco Miguel, aldeas e términos de la dicha villa, e en sus devisas e labranças.

\section{Partícula de unión}

Por ende,

\section{Citación a los interesados}

que todos los que son surqueros de las dichas heredades, que lo vayan ver apear por que después non pretendan inorançia que lo non supieron,

\section{Anuncio del número de pregón} que este es el segundo pregón.

\section{Testigos}

Testigos que fueron presentes: Diego López, regidor, e Fernand Gómez de Villarejo, e Alvar Fernáiz el moço.

\section{Diferencia de tiempo con el siguiente documento}

7 días

\section{DOCUMENTO 4}

Siete días más tarde se promulga el tèrcer pregón, idéntico en todo a los dos primeros.

1452, mayo 18, Sepúlveda

Frutos Sánchez, pregonero público de Sepúlveda, a petición de Aivar Rodríguez 
de Vellosillo, vecino de Sepúlveda, hace saber, en tercer pregón. a los que tienen heredades en Rebollar, Valdesimonte, Aldea el Pozo. Fresnillo de la Fuente, Pajarejos, Bercimuel, Grajera, Castillejo, Aldealapeña, Sigueruelo. Casla y Velasco Miguel, aldeas de Sepúlveda, que se va a proceder al deslinde de las posesiones del citado Alvar Rodríguez en dichos lugares.

CDS II, 108.

1. Partícula de unión con documento anterior

E después desto,

2. Lugar del pregón

en el dicho arraval de la dicha Sepúlvega,

\section{Data}

diez e ocho días del dicho mes de mayo del año susodicho.

4. Presencia de escribano-testigos

en presençia de mí el dicho escrivano e de los testigos de yuso escritos,

5. "I Iussio-rogatio»

por mandado del dicho alcallde e a pedimiento del dicho Alvar Rodríguez de Vellosillo,

6. Anuncio del pregón

por el dicho Frutos Sánchez, pregonero público en la dicha villa, fue dado el terçero pregón a altas bozes en esta guisa:

\section{Notificación}

Sepan todos

\section{Anuncio del propietario}

que Alvar Rodríguez de Vellosillo, vezino de la dicha villa

\section{Anuncio del deslinde}

que quiere apear todas las heredades de pan levar, e prados, e pastos, e casas, e heras, e solares que tiene en Rebollar, e en Valdesimonte, e en Aldea el Pozo, e en Fresnillo, e en Pajarejos, e en Verzimuel, e en Grajera, e en Castillejo, e en Aldealapeña, e en Sigueruelo, e Casla, e en Velasco Miguel, aldeas e término de la dicha villa, e en sus devisas e labranças. 
10. Partíiula de unión

Por ende,

11. Citación a los interesados

que todos los que son surqueros de la dicha heredad, que lo vayan ver apear por que después non pretendan inorançia,

12. Anuncio del número de pregón

que este es el terçero pregón.

\section{Testigos}

Testigos que a esto fueron presentes: Alvar Gonçález, escrivano, e Alonso Gonçález de Gil Álvarez, e Luis Pérez, vezinos de la dicha villa, e otros.

14. Diferencia de tiempo con el siguiente documento

9 días

\section{DOCUMENTO 5}

Nueve días después se promulga el cuarto y último pregón, cuyas características difieren algo de los anteriores. En primer lugar, no es habitual en los apeos sepulvedanos la celebración de este cuarto pregón. Normalmente se efectúan a lo sumo tres, el último de los cuales tiene las características que aquí comentamos. En segundo lugar, encontramos que la iussio de la autoridad, presente en los casos anteriores, ha desaparecido y que antes del anuncio del número del pregón se fija la fecha para la celebración del apeo.

\section{2, mayo 27, Sepúlveda}

Frutos Sánchez, pregonero público de Sepúlveda, a petición de Alvar Rodríguez de Vellosillo, vecino de Sepúlveda, hace saber, en cuarto pregón, a los que tienen heredades en Rebollar, Valdesimonte, Aldea el Pozo, Bercimuel, Pajarejos, Grajera, Castillejo, Aldealapeña, Sigueruelo, Casla y Velasco Miguel, aldeas de Sepúlveda, que se va a comenzar el deslinde de las posesiones del citado Alvar Rodríguez en dichos lugares el lunes primero siguiente (día 29 de mayo).

CDS II, 109.

1. Partícula de unión con documento anterior

E después desto, 


\section{Lugar del pregón}

en el dicho arraval de la dicha Sepúlvega,

\section{Data}

veinte e siete días del mes de [mayo], año susodicho.

\section{Presencia escribano-testigos}

en presençia de mí el dicho escrivano e de los testigos de yuso escritos,

\section{5. "Rogatio"}

(sigue parte de la fórmula 6 \} a pedimiento del dicho Alvar Rodríguez de Vellosillo, vezino desta dicha villa,

\section{Anuncio del pregón}

por el dicho Frutos Sánchez, pregonero público en la dicha villa, ... fue dado el quarto pregón a altas bozes en esta guisa:

\section{Notificación}

Sepan todos

\section{Anuncio del propietario}

que Alvar Rodríguez de Vellosillo, vezino desta villa

\section{Anuncio del deslinde}

que quiere apear todas las heredades de pan llevar, e prados, e pastos, e casas, e heras, e solares quel dicho Alvar Rodríguez tiene en Rebollar, e en Valdesimonte, e en Aldea el Pozo, e en Verzimuel, e en Pajarejos, e en Grajera, e en Castillejo, e en Aldealapeña, e en Sigueruelo, e en Casla, e en Velasco Miguel, aldeas e término de la dicha Sepúlvega, e en sus devisas e labranças.

\section{Partículo de unión}

Por ende,

\section{Citación a los interesados}

que todos los que son surqueros de las dichas heredades, que lo vayan a ver apear (sigue cláusula 12) por que después no pretendan inorançia,

\section{Anuncio del día del pregón}

para el lunes primero que viene e dende en adelante para cada día, 


\section{Anuncio del número de pregón}

que este es el quarto pregón.

\section{Testigos}

Testigos que a esto fueron presentes: Pero Díez, clérigo, e Luis Pérez, vezinos de la dicha villa, e Antón Garçía, del Arraval, e otros.

\section{Diferencia de tiempo con el siguiente documento} 0 días

\section{DOCUMENTO 6}

En la misma fecha que el último pregón se redacta el sexto documento. En él en presencia de la autoridad ${ }^{13}$ comparece el procurador de quien deseaba deslindar sus propiedades y expone que se han promulgado los anteriores pregones, que se ha señalado día para realizar el apeo y que los apeadores, es decir, los expertos conocedores del terreno destinados a realizar el apeo, son viejos e flacos e non los podía presentar ante el alcalde. Por ello suplica a éste que conceda licencia al escribano que redacta el documento para recibir de los mismos el juramento establecido por el procedimiento, cosa que el alcalde concede. Como en los anteriores documentos, todo el acto tiene lugar en el arrabal de la villa.

1452, mayo 27, Sepúlveda

Juan Escalante, alcalde en Sepúlveda por Diego Delisa, corregidor en la villa por el rey, otorga poder a Pedro González, escribano público del concejo, para que reciba de las personas que le presente Nuño Núñez, vecino de Sepúlveda y procurador de Alvar Rodriguez de Vellosillo, el juramento necesario para deslindar las heredades que este último posee en Fresnillo de la Fuente y Bercimuel.

CDS II, 110.

1. Partícula de unión con documento anterior

E después desto,

\section{Lugar del pregón}

en el dicho arraval de la dicha Sepúlvega,

13 Como en los documentos anteriores, un alcalde nombrado por el corregidor, pero en este caso el corregidor es ya un oficial real, por lo que la villa habría vuelto al realengo durante la promulgación de los pregones. 


\section{Data}

este dicho día, veinte e siete días del dicho mes de mayo, mes e año susodicho,

\section{Autoridad presente}

ante Juan de Escalante, alcallde en la dicha villa por el honrado Diego Deliza, corregidor en la dicha villa e en su tierra por nuestro señor el rey,

\section{Presencia escribano-testigos}

e en presençia de mí el dicho Pero Gonçález, escrivano público por el conçejo en la dicha Sepúlvega, e de los testigos de yuso escritos,

\section{Comparecencia del propietario o de su procurador}

paresçió y presente Juan, fijo de Nuño Gonçález, vezino de la dicha villa, en nombre del dicho Alvar Rodríguez de Vellosillo,

\section{Exposición 1}

e dixo que por quanto el dicho Alvar Rodríguez e él en su nonbre avían fecho dar çiertos pregones en çiertas heredades que el dicho Alvar Rodríguez tiene en el dicho Fresnillo e en Verzimuel, aldeas de la dicha villa,

\section{Exposición 2}

e avía señalado día para lo apear para este lunes primero que viene e dende en adelante para cada día,

\section{Exposición 3}

e los apeadores de quien él se entiende de aprovechar para lo apear las dichas heredades son viejos e flacos ${ }^{14}$ e non los podía traer a los presentar antel dicho alcallde,

\section{Partícula de unión}

por ende,

\section{Súplica}

dixo que pedía e pidió al dicho alcallde que diese poder a mí el dicho escrivano para que pudiese resçebir juramento en forma de los dichos apeadores.

${ }^{14}$ Se trata de una circunstancia habitual en estos documentos sepulvedanos. Los apeadores nunca suelen comparecer ante la autoridad alegando motivos de salud, ancianidad o similares a los expresados. 


\section{Disposición}

E luego el dicho alcallde dixo que oía lo que dezía e que mandava e mandó a mí el dicho escrivano que resçibiese juramento en forma de los dichos apeadores, e de cada uno dellos, quel dicho Juan en nombre del dicho Alvar Rodríguez ante mí presentase,

\section{Cesión de poder al escribano}

para lo qual dixo que me dava e dio a mí el dicho escrivano todo su poder complido.

\section{Testigos}

Testigos que a esto fueron presentes, los dichos Pero Díez, clérigo, e Luis Pérez, e Antón García, vezinos de la dicha villa, e otros.

15. Diferencia de tiempo con el siguiente documento

2 días

\section{DOCUMENTO 7}

Dos días después tiene lugar el último acto del apeo. Se celebra ya en la primera aldea en cuyas tierras existen tierras del propietario, Fresnillo de la Fuente. De nuevo comparece el procurador del interesado que presenta a las personas que se encargarán de hacer el deslinde, los apeadores, que son dos vecinos de la aldea. Acto seguido, el escribano, según había sido facultado, recibe el juramento de ambos. Después se procede finalmente al deslinde de las propiedades.

1452, mayo 29, Fresnillo de la Fuente

Deslinde de las heredades que Alvar Rodríguez de Vellosillo, vecino de Sepúlveda, posee en Fresnillo de la Fuente, aldea de Sepuilveda, hecho por Diego Martín, toquero, y por su bermano Pascual Martín, vecinos de Vellosillo, a requerimiento de Juan, bijo de Nuño González, escudero y criado del dicho Alvar Rodríguez, en presencia de Pedro González, escribano público por el concejo de Sepúlveda.

CDS II, 111.

1. Partícula de unión con documento anterior

E después desto,

2. Lugar

en el dicho lugar Fresnillo, aldea e término de la dicha Sepúlvega, 


\section{Data}

veinte e nueve días del dicho mes de mayo, mes e año susodicho,

\section{Presencia escribano-testigos}

e en presençia de mí el dicho escrivano público sobredicho, e de los testigos de yuso escritos,

\section{Comparecencia del procurador del propietario}

paresçió y presente el dicho Juan, escudero del dicho Alvar Rodríguez de Vellosillo,

\section{Disposición 1}

e dixo que presentava e presentó por apeadores para apear la dicha heredad, quel dicho Alvar Rodríguez tiene en el dicho lugar Fresnillo e en sus devisas e labranças, a Diego, toquero, e a Pascual Martín, su hermano, vezinos de la dicha aldea,

\section{Disposición 2}

de los quales e de cada uno dellos yo, el dicho escrivano, por poder e mandado del dicho Juan d'Escalante, alcallde en la dicha villa, resçibí juramento sobre la señal de la Cruz + en forma devida, ${ }^{15}$

\section{Juramento 1}

que bien e leal e verdaderamente, sin arte e sin engaño, apearían e declararían e demostrarían toda la dicha heredad, e prados, e pastos, e heras, e solares, e cada una cosa dello; ${ }^{16}$

\section{Juramento 2}

e echadas las fuerças e confusiones del dicho juramento, los dichos apeadores e cada uno dellos respondieron e dixeron «Si juro" e "Amén".

(sigue el deslinde)

${ }^{15}$ En Colección Diplomática (cit.), doc. 68, de 1416, el apeador realizó su juramento commo buen christiano.

${ }^{16}$ En Colección diplomática (cit.), doc. 93, de 1450, aparece una fórmula más completa del juramento. Entre corchetes hemos añadido algunas palabras que proceden del doc. 121 , de 1454, de la colección: ressibi juramento de los dichos ... sobre la señal de la Cruz + ten que cada uno puso su mano derecha) en forma devida, que bien e lealmente, sin arte e sin engaño e sin encubierta ninguna (e sin cobdigia), que apearán la dicha heredad e todo lo que con ello se contiene, e que nin por pérdida nin por gananģia, nin por fazer bien nin mal a ninguna persona, nin por miedo nin por favor de ninguna persona, nin por quitar a los unos nin por dar a los otros, que non dirán otra cosa sinon la verdad de todo lo que supiesen... 


\section{Testigos}

Testigos que a esto fueron presentes, los dichos Miguel Ferrández, e Miguel Sánchez, regatero, vezinos de la dicha aldea, e el dicho Juan, escudero del dicho Alvar Rodríguez, criado del dicho Alvar Rodríguez, e otros.

El deslinde propiamente dicho aparece entre las fórmulas 9 y $10 \mathrm{del}$ último documento. En él se describen un total de 117 propiedades en entradas independientes, cada una de las cuales presenta los siguientes apartados:

- Indicación del tipo de propiedad: E luego los apeadores apearon una tierralpradolcasa...

- Situación geográfica de la misma: al hanoria que dizen, çerca de la dicha aldea

- Características de la misma:çerrada de seto de enebro

- Sus límites, fijados por los nombres de los propietarios de las tierras contiguas: que ha por linderos de la una parte...

En resumen, el proceso de los deslindes sepulvedanos pasa por los siguientes actos administrativos: ${ }^{17}$

1. Petición a la autoridad (alcalde nombrado por el corregidor) por parte del interesado, que comparece personalmente, para promulgar los pregones y realizar el apeo (documento 1 ).

2. Concesión de la licencia por la autoridad para promulgar los pregones y realizar el apeo (documento 1 ).

3. Mandato u orden de la autoridad para que se realicen los pregones según la costumbre de la villa (documento 1 ).

4. Promulgación de los pregones por el pregonero público ${ }^{18}$ de la villa en el arrabal de la misma. En total se celebran cuatro pregones, aunque lo habitual en Sepúlveda es que sólo tuvieran lugar tres (documentos 2 a 5). El primer pregón tiene lugar el mismo día que los actos primero a tercero, el segundo se celebra siete días después, el tercero siete días después del anterior y el cuarto y último nueve días después del tercero.

5. Citaciones a los interesados o afectados por el deslinde para que se

17 Diremos que hemos encontrado una absoluta concordancia en este punto en todos los diplomas sepulvedanos del siglo XV que reseñan apeos de bienes rústicos (tierras, prados, viñas, majuelos etc.) y urbanos (casas, solares, pajares etc.).

${ }_{18}$ Es curioso que a, fines de la Edad Media, el oficio de pregonero estuviera vinculado al de maestro de gramática en Sepúlveda. 
den por enterados de su celebración, asistan al mismo y no puedan alegar ignorancia con posterioridad (documentos 2 a 5 ).

6. Fijación de la fecha del deslinde (documento 5).

7. Concesión por la autoridad (un alcalde) de facultad al escribano que da fe del proceso del deslinde para que pueda recibir los juramentos exigidos a los apeadores (documento 6). La concesión tiene lugar en la misma fecha que el cuarto pregón y que los actos quinto y sexto.

8. Acto de presentación de los apeadores (documento 7). Todos los actos anteriores se celebran en Sepúlveda. El presente y los siguientes tienen ya lugar en la aldea donde se va a realizar el deslinde.

9. Juramento de los apeadores (documento 7).

10. Ejecución del apeo (documento 7). Estos tres últimos actos se celebran dos días después del séptimo.

Con respecto a la autoridad que interviene en el desarrollo de todo el proceso tenemos que decir que al parecer poco importaba si era señorial o real, puesto que ya hemos aludido al cambio que se produce entre los primeros y los últimos actos del deslinde, cambio que no parece afectar de forma alguna al proceso. No faltan tampoco casos en los que la autoridad es eclesiástica ${ }^{19}$, en los que el desarrollo del apeo es similar al que hemos descrito.

Resumimos, por último, en un cuadro todos los actos que integran el el procedimiento administrativo que hemos descrito.

\begin{tabular}{cccc}
\hline ACTO & DOCUMENTO & FECHA & LUGAR \\
\hline 1. PETICIÓN & 1 & 1452, MAYO 4 & ARRABAL \\
\hline 2. LICENCIA & 1 & MAYO 4 & ARRABAL \\
\hline 3. MANDATO & 1 & MAYO 4 & ARRABAL \\
\hline 4. PREGONES & 2 & MAYO 4 & ARRABAL \\
& 3 & MAYO 11 & $\begin{array}{l}\text { ARRABAL } \\
\text { ARRABAL } \\
\end{array}$ \\
& 4 & MAYO 18 & ARABAL \\
\hline
\end{tabular}

19 Véase la nota 8. 


\begin{tabular}{cccc}
\hline ACTO & DOCUMENTO & FECHA & LUGAR \\
\hline 5. CITACIÓN & 2 a 5 & MAYO 4 a 27 & ARRABAL \\
\hline $\begin{array}{l}\text { 6. FIJACIÓN DE } \\
\text { FECHA DEL APEO }\end{array}$ & 5 & MAYO 27 & ARRABAL \\
\hline $\begin{array}{l}\text { 7. FACULTAD AL } \\
\text { ESCRIBANO }\end{array}$ & 6 & MAYO 27 & ARRABAL \\
\hline $\begin{array}{l}\text { 8. PRESENTACIÓN } \\
\text { DE APEADORES }\end{array}$ & 7 & MAYO 29) & FA FUENTE \\
\hline $\begin{array}{l}\text { 9. JURAMENTO } \\
\text { 10. EJECUCIÓN } \\
\text { DEL APEO }\end{array}$ & 7 & MAYO 29 & FRESNILLO \\
\hline
\end{tabular}

Proceso de los apeos o deslindes de Sepúlveda (siglo XV).

\section{RÉSUMÉ}

Le apeo ou deslinde de beredades c'est un type de document avec plein valeur diplomatique et juridique, même si ce fait a été ignoré longtemps par la recherche specialisée. Dans cet article on décrit comment ces documents etaient rédigés dans les sources du village medieval espagnol de Sepúlveda ( $\mathbf{x v}^{e}$ siècle). Le apeo est rappresenté par sept documents differents, emis au long d'un mois, dans lesquels un propriétaire tâche de délimiter toute sa propriété, soit il des proprietes urbaines que rurales. Dans ces documents on peut trouver dix actes juridiques: petition, licence et ordre pour faire le apeo; édition des annonces publiques et citations; fixation de la date pour la réalisation de l'apeo; concession de faculté au notaire; presentation des personnes qui devaient fair l'apeo (les apellés apeadores dans les documents); jurement et exécution du apeo.

\section{SUMMARY}

The apeo or deslinde de beredades is a type of document with complete juridic and diplomatic value, although this fact has been for a long time ignored by specialised researchers. We describe in this article the way this records are written down in the medieval spanish town of Sepúlveda (XVth century). The apeo is 
represented by seven documents, issued during almost a month, in which a proprietor tries to delimit all his possessions, so urban as rural ones (houses, fields, and so on). In this documents, ten juridical acts take place: petition, license and order to make the apeo; edition of public announcemets and citations; date fixing; conferring the faculty to the public notary; presentation of people that were in charge of making the apeo (the socalled apeadores); oath and the execution of the apeo or boundies marking. 